\title{
ON CARLSON'S DEPTH CONJECTURE
}

\author{
JAMES A. SCHAFER
}

\section{INTRODUCTION}

Let $A=\oplus_{i \geq 0} A_{i}$ be a finitely generated graded commutative $k$-algebra where $k$ is a field and $M=\oplus_{i \geq 0} M_{i}$ a finitely generated graded $k$-module.

Definition 1.1. $\omega_{A}(M)=\min \left\{\operatorname{dim} P \mid P \in \operatorname{Ass}_{A} M\right\}$. For $M=A$ denote this simply by $\omega A$.

It is well known (shown originally by Serre) that $\operatorname{depth}_{A} M \leq \omega_{A} M$. For a proof of this as well as other standard results of commutative algebra adapted to the graded commutative $k$-algebra setting a good reference is [16. Clearly $\omega_{A} M \leq$ $\operatorname{dim}_{A} M$ and so $\operatorname{depth}_{A} M \leq \omega_{A} M \leq \operatorname{dim}_{A} M$ for all finitely generated $M$.

It is quit easy to produce examples where $\operatorname{depth}_{A} M<\omega_{A} M$. However based on the work of Benson and Carlson in [5, Carlson in [7] asked whether it might not be the case that $\omega A=\operatorname{depth} A$ if $A=H^{*}(G, k)$ where $k$ is a field whose characteristic divides the order of the finite group $G$. This is obviously true if $H^{*}(G, k)$ is CohenMacaulay since $\operatorname{depth} A=\operatorname{dim} A$ in that case. Carlson showed in the same paper the conjecture is true if $\operatorname{dim} H^{*}(G, k)=2$. Except for innumerable calculations showing the conjecture was true the only other general result is one of D.J Green in 13. A basic result of Duflot 8 states that if $k$ is a field of characteristic $p>0$ then the $p$-rank of the center of $G$ is a lower bound for the depth of $H^{*}(G, k)$. Green shows that if $G$ is a $p$-group and the depth of $H^{*}(G, k)$ equals Duflot lower bound then depth $H^{*}(G, k)=\omega H^{*}(G, k)$.

Note that because of the result of Duflot if $\operatorname{dim} H^{*}(G, k)=2$ the gap between the dimension and the depth of $H^{*}(G, k)$ is at most one. Carlson's result could be interpreted to say that if the gap is less than or equal to one (and the dimension is two) then the conjecture is true. It is the aim of this paper to demonstrate the following

Theorem. Let $G$ be a finite group and $k$ a field whose characteristic divides the order of $G$. If $\operatorname{dim} H^{*}(G, k)-\operatorname{depth} H^{*}(G, k)=1$, then depth $H^{*}(G, k)=\omega H^{*}(G, k)$.

\section{Reduction to the Case $A=k\left[x_{1}, \ldots, x_{d}\right]$}

Proposition 2.1. 1) Let $f: S \rightarrow A$ be a homomorphism of Noetherian rings and suppose $A$ is finitely generated as an $S$-module. Let $M$ be a finitely generated $A$ module. Then $A s s_{S} M=f^{-1}\left(A s s_{A} M\right)$. If $P \in A s s_{A} M$ then $\operatorname{dim} P=\operatorname{dim} f^{-1} P$ and hence $\omega_{A} M=\omega_{S} M$.

2) Let $f: S \rightarrow A$ be an epimorphism of Noetherian rings and $M$ a finitely generated $A$-module. Suppose either $S$ and $A$ are local and $f\left(\mathfrak{m}_{S}\right) \subseteq \mathfrak{m}_{A}$ or $S$ and $A$ are graded commutative finitely generated $k$ algebras where $k$ is a field, $f$ is a

Date: March 24, 2021. 
graded homomorphism and $M$ a finitely generated graded module. Then depth ${ }_{S} M=$ $\operatorname{depth}_{A} M$.

3) In the graded commutative case $f$ can also be an inclusion with $A$ integral over $S$.

Proof. 1) If $Q$ is an $A$-primary in $M$ with associated prime $P=r\left(\operatorname{ann}{ }_{A} M / Q\right)$ then it is easily seen that $Q$ is $S$-primary with associated prime $f^{-1} P=r\left(\operatorname{ann}{ }_{S} M / Q\right)$. Hence if $0=\bigcap Q$ is a minimal primary decomposition of 0 as an $A$-module we obtain a minimal decomposition of 0 as an $S$-module by intesecting all those $Q$ whose associated primes have the same inverse image under $f$. If $P_{i}$ is the prime associated to the $A$-primaray module $Q_{i}$ then this gives an $S$-primary decompostion $(0)=\cap \bar{Q}_{r_{j}}$ where $\bar{Q}_{r_{j}}=\cap\left\{Q_{i} \mid f^{-1} P_{r_{i}}=f^{-1} P_{r_{j}}\right\}$. This is a primary decomposition of (0) with distinct primes and it must be minimal for if $\cap_{j^{\prime} \neq j} \bar{Q}_{r_{j^{\prime}}} \subseteq \bar{Q}_{r_{j}}$ then $\cap_{s \neq r_{j}} Q_{s} \subseteq Q_{r_{j}}$ which is impossible since $(0)=\cap Q_{i}$ is a minimal primary decomposition. The last statement follows since $S / f^{-1} P \subseteq A / P$ and the latter is finitely generated over the former.

2) If $f$ is an epimorphism and $\left\{x_{1}, \ldots, x_{k}\right\}$ is a maximal $M$-sequence in $A^{+}$, we may choose $y_{i} \in S^{+}$(of the same degree) with $f\left(y_{i}\right)=x_{i}$. Since $y_{i} m=x_{i} m$ for all $m \in M,\left\{y_{1}, \ldots, y_{k}\right\}$ is an $M$-sequence in $S^{+}$. Hence $\operatorname{depth}_{S} M \geq \operatorname{depth}_{A} M$. If $\left\{y_{1}, \ldots y_{t}\right\}$ is an $M$-sequence in $S^{+}$then since $y_{i} m=f\left(y_{i}\right) m,\left\{f\left(y_{1}\right), \ldots, f\left(y_{t}\right)\right\}$ is an $M$-sequence in $A^{+}$and so $\operatorname{depth}_{A} M \geq \operatorname{depth}_{S} M$.

3) In 12] Evens shows this is true in the strictly commutative case if $A$ is integral extension of $S$. An $M$-sequence for $M$ consists of algebraically independent elements since any $M$-sequence can be exended to a system of parameters for $M$ and these are algebraically independent, [6], theorem 2.2.7. Hence any $M$-sequence is contained in $S^{e v}$ or $A^{e v}$. Hence $\operatorname{depth}{ }_{S}^{e v} M=\operatorname{depth}_{S} M$ and therefore $\operatorname{depth}_{A} M=\operatorname{depth}_{S} M$.

\section{Modules over Regular local or $*$-LOCAl domains}

In this section, $S$ will always be a Noetherian, regular ring. By $(S, \mathfrak{m})$ we will mean a regular finitely generated graded commutative $k$-algebra over a field $k$ with $S_{0}=k$ and where $\mathfrak{m}$ is the maximal homogeneous ideal of elements of positive degree or a regular local ring and $\mathfrak{m}$ is the maximal ideal. We will just say $(S, \mathfrak{m})$ is a local ring in both cases. If $M$ is a finitely generated $S$-module, graded in the graded case, pd $M$ denotes the projective dimension of $M$ over $S$ and $H_{J}^{i}(M)$ is the local cohomology of the finitely generated module $M$ with respect to the ideal $J \subseteq S$.

Theorem 3.1. i) For finitely generated $\left.M, \min \left\{j \mid \operatorname{Ext}_{S}^{j}(M, S)\right) \neq 0\right\}=\operatorname{codim} M$.

ii) If $(S, \mathfrak{m})$ is local then $\max \left\{j \mid \operatorname{Ext}_{S}^{j}(M, S) \neq 0\right\}=p d M$.

Proof. i) $\operatorname{codim} M=\operatorname{codim}(\operatorname{ann} M), \operatorname{codim} I=\operatorname{depth} I=\operatorname{depth}(I, S)$ for all ideals $I \subseteq S$ since $S$ is Cohen-Macauley. But depth $(\operatorname{ann} M, S) \geq n$ if and only if $\operatorname{Ext}_{S}^{j}(M, S)=0$ for all $j<n$ by [9], Proposition 18.4.

The proof of ii) uses the following duality theorem for Gorenstein local rings. Proofs for the local case may be found in [15], 11.8 and for the graded $k$-algebra case in [1] 11.2.5 and 2], 3.58. $N^{\vee}$ is the Matlis dual $\operatorname{Hom}_{S}(N, E)$ where $E=E(S / \mathfrak{m})$ is the injective envelope of $S / \mathfrak{m}=k$. In the graded case $N^{\vee}$ may be identified, [2], 3.6.16, with graded Hom functor

$$
* \operatorname{Hom}_{k}(N, k) \text { where } * \operatorname{Hom}_{k}(N, k)_{s}=\operatorname{Hom}_{k}\left(N_{-s}, k\right) .
$$


Remark 3.2. In the graded case, define graded Hom, $* \operatorname{Hom}_{S}(M, N)_{t}=\{f: M \rightarrow$ $N \mid f\left(M_{u}\right) \subseteq N_{u+t}$ for all $\left.u\right\}$. If $M$ is finitely generated then $* \operatorname{Hom}_{S}(M, N)=$ $\operatorname{Hom}_{S}(M, N)$ and therefore $* \operatorname{Ext}_{S}^{*}(M, N)=\operatorname{Ext}_{S}^{*}(M, N)$ for all $N$.

Theorem 3.3. Let $(S, \mathfrak{m})$ be a Gorenstein, local ring of dimension $d$ and $M a$ finitely generated $S$-module. Then there exists a natural isomorphism

$$
H_{\mathfrak{m}}^{i} M \cong \operatorname{Ext}_{S}^{d-i}(M, S)^{\vee} .
$$

Remark 3.4. In the graded case 2] we have an isomorphism of graded modules $\left(H_{\mathfrak{m}}^{i} M\right)^{\vee} \simeq \operatorname{Ext}_{S}^{d-i}(M, S[a])$ for some $a$. Since $H_{\mathfrak{m}}^{j} M$ is Artinian and duality is an anti equivalence of the full subcategories of the category of graded $(S, \mathfrak{m})$-modules consisting of the finitely generated modules and the Artinian modules we obtain the dedsired isomorphism. If $S=k\left[x_{1}, \ldots, x_{n}\right]$ then $a=-\sum \operatorname{degree}\left(x_{i}\right)$.[2] p.140.

Proof of ii) By Auslander-Buchsbaum pd $M+\operatorname{depth} M=d$. But

$$
\operatorname{depth} M=\min \left\{j \mid H_{\mathfrak{m}}^{j} M \neq 0\right\}=d-\max \left\{e \mid \operatorname{Ext}_{S}^{e}(M, S)^{\vee} \neq 0\right\}
$$

and so

$$
\operatorname{pd} M=\max \left\{e \mid \operatorname{Ext}_{S}^{e}(M, S)^{\vee} \neq 0\right\} .
$$

But the Matlis duality functor is faithful (obvious in the graded case) and hence the conclusion.

The following result by Eisenbud, Huenke and Vasconselos will be essential.

Theorem 3.5 (11, Theorem 1.1). Let $M$ be a finitely generated module over a regular domain $S$ and set $I_{e}=$ ann $\operatorname{Ext}_{S}^{e}(M, S)$ :

(1) $\operatorname{codim} I_{e} \geq e$ and $M /\left(0:_{M} I_{e}\right)$ has no associated primes of codim e. In particular, if $P \in \operatorname{Spec} S$ and $\operatorname{codim} P=e$ then $P \in A$ ss $(M)$ iff $P \in V\left(I_{e}\right)$.

(2) If $c=\operatorname{codim} M$ then hull $(0, M)$ is the kernel of the natural map

$$
\left.\varphi: M \rightarrow \operatorname{Ext}_{S}^{c}\left(\operatorname{Ext}_{S}^{c}(M, S), S\right)\right) .
$$

If $M$ is a finitely generated $S$-module, let $(\operatorname{Ass} M)_{e}=\{P \in \operatorname{Ass} M \mid \operatorname{codim} P=e\}$.

Proposition 3.6. Let $(S, \mathfrak{m})$ be a regular domain and $M$ a finitely generated $S$ module. Then

$$
\begin{gathered}
\operatorname{Ext}_{S}^{j}(M, S)=0 \text { implies }(\text { Ass } M)_{j}=\emptyset \text { hence } \\
\max \left\{j \mid(\text { Ass } M)_{j} \neq \emptyset\right\} \leq \max \left\{j \mid \operatorname{Ext}_{S}^{j}(M, S) \neq 0\right\} .
\end{gathered}
$$

Proof. Let $P \in \operatorname{Ass} M$ be of codimension $j$. Then $P_{P}$ is an $S_{P}$-associated prime of $M_{P}$ which is non-zero since $P \in V(\operatorname{ann} M)=\operatorname{Supp} M$. Hence $S_{P} / P_{P} \subseteq M_{P}$ and $H_{P}^{0}\left(M_{P}\right) \neq 0$ since $H_{P}^{0}\left(M_{P}\right)$ is the maximal submodule $M^{\prime}$ of $M_{P}$ of finite type. $H_{P}^{0}\left(M_{P} / M^{\prime}\right)=0$ and therefore $\operatorname{depth} M_{P} / M^{\prime}>0$ and $\mathrm{pd} M_{P} / M^{\prime}<j$ by Auslander-Buchsbaum. Hence

$$
\operatorname{Ext}_{S_{P}}^{j}\left(M_{P}, S_{P}\right) \rightarrow \operatorname{Ext}_{S_{P}}^{j}\left(M^{\prime}, S_{P}\right)
$$

is an isomorphism. Since $\operatorname{dim} S_{P}=j, \operatorname{Ext}_{S_{P}}^{j}\left(M^{\prime}, S_{P}\right) \neq 0$ and therefore

$$
\operatorname{Ext}_{S_{P}}^{j}\left(M_{P}, S_{P}\right)=\operatorname{Ext}_{S}^{j}(M, S)_{P} \neq 0 .
$$

Theorem 3.7. Let $e=p d M$ and $I=I_{e}=\operatorname{ann} \operatorname{Ext}_{S}^{e}(M, S)$. Then $(A s s M)_{e} \neq \emptyset$ if and only if $H_{I}^{0}(M) \neq 0$. 
Proof. Since $e=\operatorname{pd} M,(\operatorname{Ass} M)_{f}=\emptyset$ for $f>e$. From the above result, $\operatorname{codim} I \geq e$ and $(\operatorname{Spec} S)_{e} \cap V(I)=(\text { Ass } M)_{e}$. Therefore $(\text { Ass } M)_{e}=\emptyset$ implies (is equivalent to) all associated primes $P$ of $M$ have $I \nsubseteq P$ which in turn implies $I_{P}=S_{P}$ and hence $\left(H_{I}^{0}(M)\right)_{P}=H_{I_{P}}^{0}\left(M_{P}\right)=(0)$ but this implies $H_{I}^{0}(M)=(0)$ by [E], Cor. 3.5. Conversely if $(\text { Ass } M)_{e} \neq \emptyset$ then there exists $P \in$ Ass $M$ with $I \subseteq P$. But $P_{P} \in$ Ass $S_{P} M_{P}$ and so depth $\left(S_{P}, M_{P}\right)=0$. But this is equivalent to $H_{P_{P}}^{0} M_{P} \neq(0)$ and since $H_{P_{P}}^{0} M_{P} \subseteq H_{I_{P}}^{0} M_{P}$ we have $\left(H_{I}^{0} M\right)_{P} \neq(0)$ and hence $H_{I}^{0} M \neq(0)$.

Remark 3.8. An even easier proof is to note that by [9] corollary 3.13 and [11, Ass $H_{I}^{0} M=(\operatorname{Ass} M)_{e}$.

Theorem 3.9. Let $(S, \mathfrak{m})$ be a local, regular domain and $M$ a finitely generated $S$-module with $p d M=e$. Let $I=I_{e}=$ ann $\operatorname{Ext}_{S}^{e}(M, S)$. Then the following are equivalent.

(1) $\operatorname{depth} M=\omega M$.

(2) $H_{I}^{0}(M) \neq 0$.

(3) $\operatorname{Hom}_{S}\left(\operatorname{Ext}_{S}^{e}(M, S), M\right) \neq 0$.

(4) $\operatorname{Tor}_{e}^{S}(M, M) \neq 0$.

Proof. Since pd $M=e, \operatorname{Ext}_{S}^{f}(M, S)=(0)$ if $f>e$, hence $(\text { Ass } M)_{f}=\emptyset$ for $f>e$.

(1) $\Longleftrightarrow$ (2). If $\operatorname{dim} S=s$ then $\operatorname{depth} M=s-\operatorname{pd} M=s-e$ by AuslanderBuchsbaum. On the other hand $\omega M=s-\max \{j \mid($ Ass $M) j \neq \emptyset\}$. Therefore $\operatorname{depth} M=\omega M$ if and only if (Ass $M)_{e} \neq \emptyset$ if and only if $H_{I}^{0} M \neq(0)$.

$(2) \Longleftrightarrow(3)$ Let $\bar{M}=\operatorname{Ext}_{S}^{e}(M, S)$. From [E], Proposition 18.4, for all $N$ with ann $N+\operatorname{ann} \bar{M} \neq S$

$$
\operatorname{depth}(I, N)=\min \left\{r \mid \operatorname{Ext}_{S}^{r}(\bar{M}, N) \neq 0\right\} .
$$

But ann $M \subseteq$ ann $\bar{M}$ and so

$$
\operatorname{depth}(I, M)=\min \left\{r \mid \operatorname{Ext}_{S}^{r}(\bar{M}, M) \neq 0\right\} .
$$

But from [15], 6.9, $\operatorname{depth}(I, M)=\min \left\{t \mid H_{I}^{t} M \neq 0\right\}$. Therefore

$$
\left.H_{I}^{0} M \neq 0 \Longleftrightarrow \operatorname{depth}(I, M)=0 \Longleftrightarrow \operatorname{Hom} \operatorname{Ext}_{S}^{e}(M, S), M\right) \neq 0 .
$$

(3) $\Longleftrightarrow$ (4). If $F_{*} \rightarrow M \rightarrow 0$ is a minimal free $S$-resolution of $M$ then

$$
\operatorname{Hom}_{S}\left(F_{e-1}, S\right) \rightarrow \operatorname{Hom}_{S}\left(F_{e}, S\right) \rightarrow \operatorname{Ext}_{S}^{e}(M, S) \rightarrow 0
$$

is exact and hence

$\left.\left.0 \rightarrow \operatorname{Hom}_{S}\left(\operatorname{Ext}_{S}^{e}(M, S), M\right) \rightarrow \operatorname{Hom}_{S}\left(\operatorname{Hom}_{S}\left(F_{e}, S\right), M\right)\right) \rightarrow \operatorname{Hom}\left(\operatorname{Hom}_{S}\left(F_{e-1}, S\right), M\right)\right)$

is exact. But for any finitely generated projective module $P$, there is a natural isomorphism $P \otimes M \rightarrow \operatorname{Hom}_{S}\left(\operatorname{Hom}_{S}(P, S), M\right)$ given by $x \otimes m \mapsto g$ where $g(f)=$ $f(x) m$. Hence there exists an exact sequence

$$
0 \rightarrow \operatorname{Hom}_{S}\left(\operatorname{Ext}_{S}^{e}(M, S), M\right) \rightarrow F_{e} \otimes M \rightarrow F_{e-1} \otimes M
$$

and therefore $\operatorname{Hom}_{S}\left(\operatorname{Ext}_{S}^{e}(M, S), M\right) \simeq \operatorname{Tor}_{e}^{S}(M, M)$. Hence $H_{I}^{0} M \neq 0$ if and only if $\operatorname{Tor}_{e}^{S}(M, M) \neq 0$.

Remark 3.10. In the graded case since $* \operatorname{Hom}_{S}(A, B)[a]=* \operatorname{Hom}_{S}(A[-a], B)=$ $* \operatorname{Hom}_{S}(A, B[a])$ for any $a \in \mathbb{Z}$, we have for any $a, b \in \mathbb{Z}, * \operatorname{Ext}_{S}^{j}(A, B) \neq 0$ if and only if $* \operatorname{Ext}_{S}^{j}(A[a], B[b]) \neq 0$. 
Theorem 3.11. Let $(S, \mathfrak{m})=k\left[x_{1}, \ldots, x_{n}\right]$ be a graded polynomial ring over the field $k$ and $M$ a finitely generated graded $S$-module of depth $d$. Then

$$
\text { depth }_{S} M=\omega_{S} M \text { if and only if } * \operatorname{Hom}_{S}\left(M^{\vee}, H_{\mathfrak{m}}^{d} M\right) \neq(0)
$$

where $M^{\vee}$ is the Matlis dual of $M$,

$$
M^{\vee}=* \operatorname{Hom}_{k}(M, k) \simeq * \operatorname{Hom}_{S}\left(M, E_{S}(k)\right) .
$$

Proof. By Auslander-Buchsbaum pd $M=e=n-d$ and we know depth ${ }_{S} M=\omega_{S} M$ if and only if $\operatorname{Hom}_{S}\left(\operatorname{Ext}_{S}^{e}(M, S), M\right) \neq 0$. If $\sigma=\sum$ degree $x_{i}$ then the above remarks say depth ${ }_{S} M=\omega_{S} M$ if and only if $\operatorname{Hom}_{S}\left(\operatorname{Ext}_{S}^{e}(M, S[-\sigma]), M\right) \neq 0$. Since $\operatorname{Ext}_{S}^{e}(M, S[-\sigma])$ is finitely generated ,

$$
\operatorname{Hom}_{S}\left(\operatorname{Ext}_{S}^{e}(M, S[-\sigma]), M\right)=* \operatorname{Hom}_{S}\left(\operatorname{Ext}_{S}^{e}(M, S[-\sigma]), M\right) .
$$

Since Matlis duality is an anti-equivalence of the full subcategories of graded $S$ modules consisting of finitely generated graded modules and Artinian modules $\operatorname{depth}_{S} M=\omega_{S} M$ if and only if $* \operatorname{Hom}_{S}\left(M^{\vee},\left(\operatorname{Ext}_{S}^{e}(M, S[-\sigma])\right)^{\vee}\right) \neq 0$. By local duality, 3.3, we have $\left.\operatorname{Ext}_{S}^{e}(M, S[-\sigma]), M\right)^{\vee} \simeq H_{\mathfrak{m}}^{d} M$.

\section{Finitely generated Graded $k$-Algebras and $H^{*}(G, k)$}

Theorem 4.1. Let $(A, \mathfrak{m})$ be a finitely generated graded commutative $k$-algebra and $M$ a finitely generated graded module of depth $d$. Let $M^{*}$ be the $k$-dual of $M$ with A-action given by $($ af $) m)=f(a m)$. Then depth ${ }_{A} M=\omega_{A} M$ if and only if $* \operatorname{Hom}_{A}\left(M^{*}, H_{\mathfrak{m}}^{d} M\right) \neq(0)$.

Proof. Let $\pi:\left(S, \mathfrak{m}_{S}\right)=\left(k\left[x_{1}, \ldots, x_{n}\right],\left(x_{1}, \ldots, x_{n}\right)\right) \rightarrow(A, \mathfrak{m})$ be an epimorphism. By 2.1 depth $_{A} M=\omega_{A} M$ if and only if $\operatorname{depth}_{S} M=\omega_{S} M$ and this occurs if and only if $\operatorname{Hom}_{S}\left(M^{\vee}, H_{\mathrm{m}_{S}}^{d} M\right) \neq(0)$. Now by change of rings for local cohomolgy there is a canonical isomorphism $H_{\mathfrak{m}_{S}}^{i} M \simeq H_{\mathfrak{m}}^{i} M$ since $\pi\left(\mathfrak{m}_{S}\right)=\mathfrak{m}$. If the $A$-module $H_{\mathfrak{m}}^{i} M$ is given an $S$-module structure by means of $\pi$ then this isomorphism is an isomorphism of $S$-modules. Since $M^{\vee} \simeq M^{*}$ and the $A$ module and $S$-module structures on $M^{*}$ correspond under $\pi$ we can conclude that $\operatorname{depth}_{A} M=\omega_{A} M$ if and only if $\operatorname{Hom}_{S}\left(M^{*}, H_{\mathfrak{m}}^{d} M\right) \neq(0)$ where both $M^{*}$ and $H_{\mathfrak{m}}^{d} M$ are $A$-modules which are $S$-modules via $\pi$. Since $\pi$ is an epimorphism $\operatorname{Hom}_{S}\left(M^{*}, H_{\mathfrak{m}}^{d} M\right)=\operatorname{Hom}_{A}\left(M^{*}, H_{\mathfrak{m}}^{d} M\right)$.

Corollary 4.2. Suppose $A=H^{*}(G, k)$ then depth $A=\omega A$ if and only if there exists a non-zero graded $H^{*}(G, k)$-map, $\lambda: H_{*}(G, k) \rightarrow H_{\mathfrak{m}}^{\text {depth } A}\left(H^{*}(G, k)\right)$ where $H_{*}(G, k)$ is an $H^{*}(G, k)$-module via cap product.

Proof. $\left(A^{*}\right)_{s}=\operatorname{Hom}_{k}\left(A^{-s}, k\right) \simeq H_{-s}(G, k)$ and it is well known that the action of $A$ on $A^{*}$ is the cap product.

Theorem 4.3. Let $G$ be a finite group and $k$ a field whose characteristic divides the order of $G$. If $\operatorname{dim} H^{*}(G, k)$-depth $H^{*}(G, k)=1$ then depth $H^{*}(G, k)=\omega H^{*}(G, k)$.

Proof. In [14] and again from a more algebraic aspect in [3] and [5] a convergent spectral sequence $\left\{E_{r}^{p, q} \mid p \geq 0, r \geq 2\right\}$ of $H^{*}(G, k)$-modules is developed with $E_{2}^{p, q}=H_{\mathfrak{m}}^{p, q} H^{*}(G, k)$ where $\left.H_{\mathfrak{m}}^{p, q} H^{*}(G, k)\right)$ is the degree $q$ part of $H_{\mathfrak{m}}^{p}\left(H^{*}(G, k)\right)$ and converging to $H_{q-p}(G, k)$. That is there is an decreasing filtration $F^{p} H_{*}(G, k)$ with $E_{\infty}^{p,-q} \simeq F^{p}\left(H_{q-p}(G, k) / F^{p+1} H_{q-p}(G, k)\right.$. Grothendieck's Vanishing Theorem says $E_{2}^{p, q}=(0)$ if $p<\operatorname{depth} H^{*}(G, k)$ or $p>\operatorname{dim} H^{*}(G, k)$ and that both $E_{2}^{\text {depth,* }}$ 
and $E_{2}^{\text {dim,* }}$ are non-zero. There is a graded $H^{*}(G, k)$ edge homomophism $\nu$ : $H_{*}(G, k) \rightarrow E_{2}^{\text {depth }, *}=H_{\mathfrak{m}}^{\text {depth }, *}\left(H^{*}(G, k)\right)$ whose image is $E_{\infty}^{\text {depth }, *}$. Hence if $E_{\infty}^{\text {depth }, *} \neq(0)$ we have a non-zero graded $H^{*}(G, k)$-homomorphism

$$
\nu: H_{*}(G, k) \rightarrow H_{\mathfrak{m}}^{*} H^{*}(G, k)
$$

and hence depth $H_{*}(G, k)=\omega H_{*}(G, k)$. If $\operatorname{dim} H_{*}(G, k)-\operatorname{depth} H_{*}(G, k)=1$ this spectral sequence has only two non-zero columns and so $E_{2}=E_{\infty}$. Grothendieck's Theorem gives the result.

\section{REFERENCES}

[1] M.P. Brodmann, R.Y. Sharp, Local Cohomology, Cambridge Studies in Advanced Mathematics 136, Cambridge University Press, 1997.

[2] W. Bruns, J. Herzog, Cohen-Macaulay Rings, Cambridge Studies in Advanced Mathematics 39, Cambridge University Press, 1993.

[3] D.J. Benson, Dickson invarints, regularity and computations in group cohomology, Illinois Journal of Mathematics, 48, (2004), 171-197.

[4] D.J. Benson, J.F. Carlson, Products in negative cohomology, Journal of Pure and Applied Algebra, 82, (1997), 107-129.

[5] D.J. Benson, Commutative algebra in the cohomology of groups, Lecture notes for MSRI Commutative Algebra Conference, 2002.

[6] D.J. Benson, Polynomial Invariants of Finite Groups, Oxford University press, 1993.

[7] J.F. Carlson, Depth and transfer in cohomology of groups, Math. Z. 218 (1995), 461-468.

[8] J. Duflot, Depth and equivariant cohomology, Comm. Math. Helv. 56, (1981), 627-637.

[9] D. Eisenbud, Commutative Algebra with a View Toward Algebraic Geometry, Graduate Texts in Mathematics 150, Springer-Verlag, 1994.

[10] D. Eisenbud, The Geometry of Syzgies, Graduate Texts in Mathematics 229, Springer-Verlag, 2005.

[11] D. Eisenbud, C. Hunke, W. Vasconselos, Direct methods for primary decomposition, Invent. Math. 110, (1992), 207-235.

[12] L. Evens, The Cohomology of Groups, Oxford Science Publications, 1991.

[13] D.J. Green, On Carlson's depth conjecture in the cohomology of groups, Math. Z. 244, (2003), 711-723.

[14] J.P.C. Greenlees, Commutative algebra in group cohomology, Journal of Pure and Applied Algebra, 98, (1995), 151-162.

[15] M. Hochster, Local Cohomology, Lecture Notes, University of Michigan.

[16] M. Poulsen, Depth, detection and associated primes in the cohomology of groups, Thesis, University of Copenhagen, 2007.

Department of Mathematics

UNIVERSITY OF MARYLAND

College Park, Maryland 20742

E-mail address: jas@math.umd.edu 